\title{
Mild cognitive impairment: Conceptual, assessment, ethical, and social issues
}

\author{
Perla Werner' \\ Amos D Korczyn² \\ 'Department of Gerontology, Faculty \\ of Social Welfare and Health Sciences, \\ University of Haifa, Haifa, Israel; \\ ${ }^{2}$ Sieratzki Chair of Neurology, \\ Department of Neurology, Tel Aviv \\ University, Ramat-Aviv, Israel
}

\begin{abstract}
Mild cognitive impairment (MCI) is defined as a condition characterized by newly acquired cognitive decline to an extent that is beyond that expected for age or educational background, yet not causing significant functional impairment. The concept of MCI has received considerable attention in the literature over the past few years, and aspects related to its definition, prevalence, and evolution have been extensively studied and reviewed.

Here we attempt to synthesize the implications of the current status of this entity, focusing on the conceptual, methodological, and, in particular, the social and ethical aspects of MCI which have attracted less attention. We discuss the weaknesses of the concept of MCI, which is heterogeneous in etiology, manifestations, and outcomes, and suggest that the emergence of the syndrome at this stage reflects industrial interests related to possible development of drugs for this disorder. On the other hand, the formal diagnosis of MCI, with its implications that the person may develop dementia, may have a grave impact on the psychological state of the individual, at a stage when prediction of outcome is tenuous and possibilities of useful interventions are meager. We present suggestions for the direction of future research in these areas.
\end{abstract}

Keywords: mild cognitive impairment, assessment issues, ethical issues, social issues, dementia

\section{Introduction}

Scientific and clinical research in the area of Alzheimer's disease (AD) during the last years shifted their focus to early diagnosis and especially to the transitional phase between normal aging and dementia. Mild cognitive impairment (MCI) is presently used to define this early phase (Petersen 1995; Petersen et al 2001).

Findings regarding the prevalence of MCI are few and inconsistent, probably due to the use of different definitional criteria and different sampling and assessment methods (for reviews see Bischkopf et al 2002; Portet et al 2006). Population-based studies showed prevalence rates ranging from 3\% to 19\% (Frisoni et al 2001; Ritchie and Artero 2001; Hanninen et al 2002), with higher rates associated with increasing age and lower education. Discrepancies are also reflected in studies examining conversion rates from $\mathrm{MCI}$ to $\mathrm{AD}$. It has been reported that approximately $2 \%$ to $25 \%$ of those carrying the diagnosis of MCI converted to AD per year (Bowen et al 1997; Kluger et al 1999; Petersen et al 1999, 2001). Using a mathematical model based on assumptions regarding $\mathrm{AD}$ prevalence rates and conversion rates from $\mathrm{MCI}$ to $\mathrm{AD}$, Yesavage and colleagues (2002) predicted that MCI prevalence increases from $1 \%$ at age 60 to $42 \%$ at age 85 . However, in this study, MCI was only defined as pre-AD. Inclusion of other MCI subtypes will of course inflate these figures.

The ongoing growth of the elderly population increases the need to further our understanding about MCI as a prodrome to dementia, about its identification and neuropathologic correlates, and pharmacological and behavioral treatment approaches. This review attempts to synthesize the current research in the area of MCI and its 
implications on conceptual, methodological, social, and ethical aspects which have attracted less attention. It is not intended to be an exhaustive nor systematic review, but to discuss and focus on several important nonmedical issues associated with this state.

\section{Conceptual issues}

The first step towards the development of a coherent body of research is to have a clear and explicit definition at the construct level, that is, to delineate the concept under study (Cohen 1991). In this section, we will therefore summarize and discuss the conceptual development and controversies related to the term $\mathrm{MCI}$.

Multiple terms have been used to describe subclinical cognitive deficits (for a more comprehensive description of the terminology developments see Petersen (2004) and Dierckx et al (2007)). These included "benign senescent forgetfulness" (BSF), proposed in the early 1960s by Kral (1962) and "age-associated memory impairment" (AAMI), introduced and adopted by the National Institute of Mental Health in the late 1980s (Crook and Larrabee 1991). Despite their contribution as heuristics for the development of research, these terms were limited by the lack of precision of the concepts, and both were regarded as variants of normal aging. Aging-associated cognitive decline (AACD) was suggested to overcome these criticisms (Levy 1994).

The prevailing term today to describe the transitional phase between normal aging and dementia is MCI. Initially, the diagnostic criteria for MCI included: 1) memory complaints, preferably corroborated by an informant; 2) objective memory impairment, beyond that expected for age and education; 3 ) normal general cognitive functioning; 4) intact activities of daily living; and 5) not being demented (Petersen and Morris 2003). Lately, the concept of MCI has been expanded to address observed clinical heterogeneity, and two subtypes were included: amnestic (consisting memory impairment) and nonamnestic (impairment in other cognitive domains) (Winblad et al 2004).

In spite of the considerable refinement in the definition of MCI, several clarifications might still be required in order for MCI to be considered as a helpful concept. For example, although the definition of AD includes the exclusion of other central nervous system conditions that could cause cognitive decline, such as Parkinson's disease and strokes, and the exclusion of systemic conditions such as hypothyroidism or vitamin B12 deficiency (as though these could not coexist with $\mathrm{AD}$ ), the definition of MCI requires the exclusion of dementia only. On a syndromic level, this is quite appropriate, since mild cognitive decline may herald other types of dementia and can occur in other brain diseases, such as multiple sclerosis. While this at the same time marks the limitations of the concept as a transitional state to AD in aging people, it should be a sign demanding explanation and possibly treatment.

Moreover, there is a need to limit the definition of MCI to newly developed cognitive decline, in order to exclude cases who might present cognitive impediments as a result of long term limitations, such as persons suffering from mental retardation or brain trauma. This obvious limitation is not included in the existing definitions (Petersen et al 2001; Ritchie and Artero 2001), but mentioned later (Portet et al 2006).

The therapeutic implications of the diagnosis of MCI should be clear. Attempts to treat MCI as if it were incipient AD, using cholinesterase inhibitors, have failed. This may not be surprising if the endpoint was conversion to dementia, since the drugs available for AD merely provide symptomatic benefit and do not slow disease progression. This situation will hopefully change once disease-modifying therapies are developed. However, at present, the heterogeneous nature of MCI should alert the clinician in each individual case to attempt to reach an etiological diagnosis that may direct therapy (eg, for depression, vascular brain disease or vitamin deficiency).

Finally, it should be stressed that MCI nosologically is not a unique entity: it is rather heterogeneous. It may carry a grave prognosis, such as becoming demented within a short time (2\%-25\% annual conversion to AD), although many people remain stable, deteriorate slowly, and a surprising number even improve spontaneously (Bowen et al 1997; Kluger et al 1999; Petersen et al 1999; Ganguli et al 2004). This again points to the difficulty of using a complaint, or a symptom, as the main diagnostic tool. The attempt to define those people who are at a prodromal stage to the development of dementia, will probably only be firmly established once biological markers of incipient dementia are identified. It is now accepted that significant AD changes can occur in the human brain prior to the emergence of symptoms (Price and Morris 1999). If this is the case, it is likely that biological markers could indeed be found.

Although it is claimed that apolipoprotein E4, neuroimaging, and neuropsychological testing may give some guidance as to who may progress more rapidly, there are no good indicators as to who will or will not convert, and this of course has important implications on whether the diagnosis should be revealed to the subject or not (see Ethical issues below).Thus, viewing MCI as "very mild AD" is erroneous 
and counterproductive (Morris 2006). If we know it to be $\mathrm{AD}$, this is the name that should be used. It seems that the prevalent use of the term is employed both to cover our ignorance at not being able to secure a definite diagnosis, as well as sometimes to provide patients with an easier-toswallow pill.

The emergence of the concept of MCI at this stage is not coincidental. Its creation and popularity are related to obvious industrial and economic interests. In the past, attempts to develop drugs for subjects with AAMI met stiff resistance from authorities such as the FDA, primarily based on the assumption that drugs should only be used to combat disease, whereas conditions such as "normal" aging should not be a target for drug manipulation. The term MCI, used to indicate a transitional state between normal aging and $\mathrm{AD}$, serves the political purpose of defining it as an abnormal state and therefore a legitimate target for drug manipulation, carrying important economic implications. Until these discrepancies are solved, debates about the usefulness of the concept will continue (Palmer and Winblad 2007; Petersen 2007; Whitehouse 2007).

\section{Assessment issues}

Assessment issues regarding MCI are still in developmental stages (for a recent review on this topic see Gauthier et al 2006). The latest extended definition of MCI calls for assessing first the person's cognition function. This can usually be accomplished by taking a clinical history and performing a mental status exam, possibly complemented by the use of neuropsychological testing, including memory tests and possibly tests for other cognitive domains. Finally, the etiology of the symptoms should be elucidated based on clinical evaluation, laboratory testing and neuroimaging studies.

Many of these stages are still subject to debate. For example, there are no details or guidance regarding the memory instrument to be used. Memory instruments vary in their psychometric characteristics, such as sensitivity and specificity (Werner 2001), and so persons meeting the definition of MCI by one instrument might be excluded if another is used. Also, the degree of dysfunction in memory test has not been defined. The 1.5 standard deviation value frequently given refers to the group value and should not be applied to an individual. In principle, any value is not appropriate, since AD develops insidiously and any chosen value is arbitrary. Moreover, it does not refer to the premorbid level of the subject. Regarding nonamnestic MCI, the confusion seems to be even larger, since no suggestions are provided as to which cognitive domains should be explored and how these should be tested.

Regarding neuropsychological tests, these should complement and not replace clinical assessment and should be brief in order to be of practical use and also in order to avoid fatigue in the elderly person. Because different domains might first be affected in a population with incipient dementia, a test which examines more than a single functional domain might be needed. One possible solution is to use self-administered, computerized neuropsychological tests (Aharonson et al 2004).

Finally, neuroimaging (such as computerized tomography and magnetic resonance imaging) and biomarkers (such as high cerebrospinal fluid [CSF] tau values, low CSF beta amyloid (Hansson et al 2006), and the presence of apolipoprotein E gene 4 allele (Treves et al 1996; Petersen et al 2001)) are helpful tools in the diagnosis of MCI (Petersen et al 2001). However, these markers are expensive and time consuming, and presumably only relevant to $\mathrm{MCI}$ in the context of incipient AD.

In summary, the last years were characterized by considerable advances in the conceptualization and assessment aspects of cognitive decline in old age (Winblad et al 2004). These advances have, however, elicited ethical and social issues. Their importance will be discussed in the following sections.

\section{Ethical issues}

Ethical issues related to dementia have received increased attention during the last decade (Post and Whitehouse 1995; Post 2000). As knowledge around MCI expands, researchers, clinicians and the lay public will be confronted with ethical issues in this area as well. In this section, we will discuss several of these issues.

\section{Disclosing the diagnosis}

One of the main ethical issues concerns the disclosure of the diagnosis of a disease to the person and his/her family. This issue involves the dilemma between the patient's right to know and withholding the information in order to avoid distress and harm (Baldwin et al 2003).

Although empirical data in the area of MCI are very limited, findings for AD may trigger further research and open the debate about the dilemmas involved in informing persons about a diagnosis of MCI. The benefits about revealing a diagnosis of $\mathrm{AD}$ include allowing patients to be involved in decision-making processes regarding their future, respecting their autonomy, and providing relief by putting a "label" to 
their symptoms (Maguire 2002; Pinner and Bouman, 2002). Pitfalls for disclosing the diagnosis to patients include concerns about precipitating fear and distress, reducing hope and positive thinking, and inducing depression and even suicidal ideation (Johnson et al 2000; Pucci et al 2003). It should be stressed that these hypothetical negative consequences were rarely supported by empirical data in $\mathrm{AD}$ (Heal and Husband, 1998; Jha et al 2001; Holroyd et al 2002; Husband 2002; Vernooij-Dassen et al 2003), and are still awaiting empirical confirmation in MCI. The situation in MCI is more complex because MCI is not identical to incipient dementia and not all cases necessarily progress (for a discussion on "reversible MCI", see de Rotou et al 2005). Thus, uncertainty concerning the prognosis in $\mathrm{MCI}$ is more prolonged than in $\mathrm{AD}$ or other dementing disorders.

It is important to stress again that not all cases diagnosed as MCI will progress (Brooks 2007), and this should be conveyed clearly to the subject, who must not be left with the impression that MCI is identical with incipient dementia. This point is particularly valid in sites where there are fewer experts who are knowledgeable about the complexities of the diagnosis of MCI, notably in Eastern Europe and Asia.

In sum, there is a need to accumulate more data to assess whether the disclosure of a diagnosis of MCI will be accompanied by increased fears, concerns, and feelings of helplessness, or whether it would help the subject and his/her family to prepare for the future. Other questions awaiting further research include the clinical practice to be used for disclosing the diagnosis, whether the label of MCI should be used or not, whether the diagnosis of MCI should be accompanied by driving restrictions, etc.

\section{Stigmatization}

Another aspect we should consider when weighing the benefits and problems of disclosing the diagnosis of MCI is whether this will affect the way the subject is viewed by others - in other words, whether a diagnosis of MCI would be accompanied by stigmatization or not.

While it is well known that stigmatization affects the lives of demented persons and their caregivers (Benbow and Reynolds 2000; Jolley and Benbow 2000; Vernooij-Dassen et al 2003), empirical research related to MCI is still scant.

A public opinion poll conducted on behalf of the Alzheimer's Society of Canada among adult Canadians (Alzheimer's Society of Canada 2003), showed that as many as $81 \%$ of the 1,000 participants felt they would be looked upon or treated differently if others knew they were diagnosed with AD. Findings of a survey of over 1,700 participants, aimed at assessing British adults' opinion about those with seven mental illnesses (severe depression, panic attacks, schizophrenia, eating disorders, alcohol addiction, drug addiction, and dementia), found that the highest percentage of participants perceiving that the patient would feel that he or she was being looked upon differently related to dementia (61\%) (Crisp et al 2000). It should be noted that while neither one of these surveys provided information about whether "being looked upon differently" related to positive or negative reactions, two recent studies found that lay persons relate to a person with $\mathrm{AD}$ with more positive than negative emotions (Wadley and Haley 2002; Werner and Davidson 2004).

The question facing us today is whether similar findings will be associated with a diagnosis of MCI. Would a person who has been given a diagnosis of MCI be "looked upon differently"? Would this label affect the diagnosed person's social functioning? Quality of life? Would his/her relatives be affected as well by the diagnosis? If the label of MCI becomes associated more with disease than with normal aging (Whitehouse and Juengst 2005), stigmatic views might prevent persons with $\mathrm{MCI}$ and their family members to seek appropriate help in an effort to conceal the problem. These issues are especially important since persons with MCI might still be actively involved in work and family roles.

\section{Social issues}

Despite the ongoing conceptual debate regarding MCI, the increase of the aging population together with the advances in the diagnosis of early dementia or MCI, will be accompanied also by social challenges, such as cost-related issues, and the development of appropriate services for persons with a diagnosis of MCI and their relatives. Although some of the services suggested in this section are associated with increased resources, it is our belief that the body of knowledge about MCI requires society to adapt existing services and/or develop new ones for this population.

\section{Economic issues}

Numerous studies in different countries have shown that the economic costs associated with the formal and informal care of AD are very high (Gray and Fenn 1993; Hux et al 1998; Coon and Edgerly 1999; Taylor and Sloan 2000; Beeri et al 2002), and that early diagnosis of the disease is associated with considerable reductions in costs (Taylor and Sloan 2000; Leifer 2003), especially in the costs of long term care services. However, as stated by Wimo and Winblad (2003) 
it is not known to what extent results regarding $\mathrm{AD}$ can be extrapolated to MCI.

With the developments in the diagnosis and treatment of MCI, increased efforts should be invested to predict the short- and long-term costs specifically associated with this diagnosis. Comas-Herrera and colleagues (2003), in a study aimed at making projections regarding the economic demands associated with cognitive impairment, found that an improved diagnosis of dementia and MCI, and a decline of $1 \%$ per year in the prevalence of moderate to severe cognitive impairment, will be accompanied by a decline on the percentage of expenses for long term care services from the Gross Domestic Product for the next 30 years. What this report fails to consider is the fact that reductions in long term care costs might be accompanied by an increase in community-based services aimed at helping persons with MCI and their relatives. Efforts should then be invested not only in the development of social services answering to the needs of those diagnosed with MCI, but also in prevention programs against the development of $\mathrm{MCI}$ (ie, primary prevention; Wimo and Winblad 2003), or its transformation to AD (ie, secondary prevention).

\section{Development of services for those with a diagnosis of $\mathrm{MCl}$}

Advances in the diagnosis of MCI have to be accompanied by a reorganization of existing services, and by the development of new services for subjects with MCI and their relatives. Some of these efforts might concentrate on expanding existing programs for elderly persons in the early stages of dementia to those diagnosed with MCI. Two such services are support groups for persons in the first stages of dementia and memory training programs.

In the last few years, we have witnessed an increase in the development of services for persons in the initial stages of dementia. The use of support groups for elderly persons in the first stages of AD started to develop in the middle to late 90's (Yale 1995; Goldsilver and Grunier 2001). Several evaluation studies showed them to be beneficial to the participants, who expressed satisfaction especially with the information and knowledge they received regarding the disease and its treatment (Chung 2001; Goldsilver and Grunier 2001; Werner 2003). Based on these findings, the use of support groups for persons with MCI should be considered. Such programs are also useful against the effects of stigmatization on the individual, and may include the provision of information regarding MCI and its progression, as well as information regarding life style changes associated with secondary prevention in persons with MCI, such as physical activity and nutritional aspects (Rosenberg et al 2006; van Uffelen et al 2007).

Another service to consider is the expansion of memory training or memory enhancement programs. These are aimed at maintaining or improving memory skills in healthy elderly persons (Scogin and Bienias 1988; Lachman et al 1992; Andrews et al 1996) by relying on a variety of cognitive techniques, such as imagery, as well as on noncognitive techniques such as relaxation. Although evidence about their effectiveness is inconsistent (McKitrick et al 1992; Sandman 1993; Floyd and Scogin 1997; Werner 2000), recent studies showed that cognitive rehabilitation might be a useful program in persons with MCI (Talassi et al 2007). Therefore, efforts to develop memory enhancement programs for persons with MCI should be expanded and their results evaluated. Such programs should be accompanied by the diffusion of knowledge about MCI, its prognosis and treatment, as well as the provision of information about the available services in the community.

Future interventions should concentrate also on developing education strategies for healthcare providers (especially for primary care providers such as family physicians, nurses, and social workers), as well as for the lay public. Recent studies assessing the lay public's knowledge about AD found that levels of knowledge are negatively associated to age (Kahana et al 2003; Werner 2004, 2005). Thus, health education programs should be aimed specifically to the needs of the older section of the population. Given the relatively low computer literacy of this group, education programs should try to reach elderly persons through more conventional sources, especially care providers, and materials published in the general media, such as television and newspapers.

\section{Development of social programs and initiatives for the prevention of $\mathrm{MCl}$}

Marked advances have been made lately in the identification of risk factors for the development of AD. Attention to clinical risk factors, such as hypertension and hyperlipidemia, have been shown to reduce or delay the incidence of dementia (Korczyn 2002). Social factors, such as high education, increased psychosocial networking, and participation in leisure activities, have also been associated with a reduced risk of dementia (Gwyther 1997; Kahana et al 2003; Seidler et al 2003; Verghese et al 2003). It is unknown as yet to what extent attention to these factors could delay the conversion of MCI to AD. Nevertheless, given these advances, it is not too soon to implement medical and social interventions for 
the primary prevention of MCI. These might include the allocation of resources for the development and conduction of information campaigns stressing the benefits of participation in appropriate health behaviors as well as in leisure activities. Additionally, efforts should be invested in training professionals not only in the identification of MCI, but also in providing adequate information and guidelines regarding its prevention and consequences. Individuals diagnosed as MCI could benefit from frank discussion of the implications of the diagnosis. Particularly in those suspected of harboring incipient dementia, discussion of writing a will and instructions about participation in research on demented individuals, prolongation of life and consent to autopsy are some obvious steps that should preferably be done prior to the development of frank dementia.

\section{Development of services for family members of persons with $\mathrm{MCl}$}

Although still limited, studies regarding burden among spouses of persons with MCI found elevated levels of care giving burden (Garand et al 2005). In consideration of these findings, efforts should be invested in developing and assessing the effectiveness of interventions geared towards the specific needs of this population.

\section{Concluding comments}

In recent years, we witnessed a growing interest in the topic of MCI. The aim of the present paper was to examine critically some conceptualization, assessment, ethical, and social issues involved, which might suggest the direction and emphasis of future research.

Although research on MCI is beyond its early stages, researchers and clinicians continue debating the usefulness of the concept. Consequently, academically led discussion groups should continue to meet and discuss the concept. Furthermore, there is need to expand the areas of research to concentrate not only on the clinical aspects of the concept but also on its ethical and societal aspects.

Overall, there is need to recognize that MCI is not a disease, but rather a syndrome. Some subjects may actually suffer from depression; others from anxiety, still others from vascular brain disease and many have prodromal AD. As a result of this, increased efforts should be made to identify the etiology of the disorder in each individual. Until a clearer understanding is achieved, rational therapy cannot be provided.

Future efforts should also be invested in the development of hypotheses examining predictors of MCI, as well as outcomes. Predictor variables may include biological and genetic factors, but also social and cultural ones. Outcome variables may include personal adjustment and mental health variables, such as depression, life satisfaction and well-being, as well as society-level outcome variables, such as cost and policy issues.

Special attention should be paid to the perspectives and insights of the persons diagnosed with MCI (Werner 2004). How does a diagnosis of MCI affect their self-esteem, anxieties and concerns? What is the effect on their relationships with family members and formal caregivers, if any (JoostenWein Banningh et al 2007)? How is their independence and functioning in social roles (such as worker, employer, spouse, parent, or grandparent) impacted?

Methodologically, there is a need to expand the research on the development and validation of reliable instruments for the assessment of MCI and particularly predicting future changes. This should be accompanied by training healthcare providers in the administration of these instruments, in order to attain a reliable diagnosis in the clinical setting, and not only in research settings. In addition, longitudinal studies aimed at examining changes along time should be encouraged.

Finally, educational efforts should be expanded and assessed systematically. These include educating lay persons regarding $\mathrm{MCI}$, and the importance of an early diagnosis of memory and other cognitive problems, as well as educating health care professionals.

\section{References}

Aharonson V, Korczyn AD. 2004. Human-computer interaction in the administration and analysis of neuropsychological tests. Comput Methods Programs Biomed, 73:43-53.

Alzheimer's Society of Canada. 2003. Stigma and Alzheimer's disease media kit: Public opinion poll summary. [Online]. Accessed March 19, 2003. URL: http://www.alzheimer.ca/english/media/stigma03poll.htm.

Andrews DG, Kinsella G, Murphy M. 1996. Using a memory handbook to improve everyday memory in community-dwelling older adults with memory complaints. Exp Aging Res, 22:305-22.

Baldwin C, Hughes J, Hope T, et al. 2003. Ethics and dementia: Mapping the literature by bibliometric analysis. Int $J$ Geriatr Psychiatry, $18: 41-54$

Beeri M, Werner P, Davidson M, et al. 2002. The cost of Alzheimer's disease in Israel. Alzheimer Dis Assoc Disord, 16:73-80.

Benbow SM, Reynolds D. 2000. Challenging the stigma of Alzheimer's disease. Hosp Med, 61:174-7.

Bischkopf J, Busse A, Angermeyer MC. 2002. Mild cognitive impairment - a review of prevalence, incidence and outcome according to current approaches. Acta Psychiatr Scand, 106:403-14.

Bowen J, Teri L, Kukull W, et al. 1997. Progression to dementia in patients with isolated memory loss. Lancet, 349:763-5.

Chung, JCC. 2001. Empowering individuals with early dementia and their carers: An exploratory study in the Chinese context. Am J Alzheimers Dis Other Demen, 16:85-8. 
Cohen, BP. 1991. Developing Sociological Knowledge: Theory and Method. Nelson Hale: Chicago.

Comas-Herrera A, Wittenberg R, Pickard L, et al.: MRC-CFAS. 2003. Cognitive impairment in older people: Its implications for future demand for services and costs. Executive summary of the Alzheimer's Research Trust [online]. Accessed March 19, 2003. URL: http://www.mrc-cfas. cam.ac.uk/cfas.

Coon, DW, Edgerly, ES. 1999. The personal and social consequences of Alzheimer's disease. Genet Test, 3:29-36.

Crisp AH, Gelder MG, Rix S, et al. 2000. Stigmatization of people with mental illnesses. Br J Psychiatry, 177:4-7.

Dubois B, Albert ML. 2004. Amnestic MCI or prodromal Alzheimer's disease? Lancet Neurol, 3:246-8.

Crook TH 3rd, Larrabee, GJ. 1991. Diagnosis, assessment and treatment of age-associated memory impairment. J Neural Transm Suppl, 33:1-6.

De Rotrou J, Wenisch E, Chausson C, et al. 2005. Accidental MCI in health subjects: a prospective longitudinal study. Eur J Neurol, 12:879-85.

Dierckx E, Engelborghs S, de Raedt R, et al. 2007. Mild cognitive impairment: what's in the name? Gerontology, 53:28-35.

Floyd, M. Scogin, F. 1997. Effects of memory training on the subjective memory functioning and mental health of older adults: A meta-analysis. Psychol Aging, 12:150-66.

Frisoni GB, Fratiglioni L, Fastbom J, et al. 2001. Mild cognitive impairment in the population and physical health data on 1,435 individuals aged 75-95. J Gerontol, 55:322-8.

Furst M, Aharonson V, Levine RA, et al. 2000. Sound lateralization and interaural discrimination. Effects of brainstem infarcts and multiple sclerosis lesions. Hear Res, 143:29-42.

Ganguli M, Dodge HH, Shen C, et al. 2004. Mild cognitive impairment, amnestic type: An epidemiologic study. Neurology, 63:115-21.

Garand L, Dew MA, Eazo LR, et al. 2005. Caregiving burden and psychiatric morbidity in spouses of persons with mild cognitive impairment. Int $J$ Geriatr Psychiatry, 20:512-22.

Gauthier S, Reisberg B, Zaudig M, et al. 2006. Mild cognitive impairment. Lancet, 367:1262-70.

Goldsilver PM, Grunier MR. 2001. Early stage dementia group: An innovative model of support for individuals in the early stages of dementia. Am J Alzheimers Dis Other Demen, 16:109-14.

Gray A, Fenn P. 1993. Alzheimer's disease: The burden of the illness in England. Health Trends, 25:31-7.

Gwyther LP. 1997. The perspective of the persons with Alzheimer's disease: Which outcomes matter in early to middle stages of dementia? Alzheimer Dis Assoc Disord, 11:18-24.

Hanninen T, Soininen H. 1997. Age-associated memory impairment: Normal aging or warning of dementia? Drugs Aging, 11:480-9.

Hanninen T, Hallikainen M, Tuomainen S, et al. 2002. Prevalence of mild cognitive impairment: A population-based study in elderly subjects. Acta Neurol Scand, 106:148-54.

Hansson O, Zetterberg H, Buchhave P, et al. 2006. Association between CSF biomarkers and incipient Alzheimer's disease in patients with mild cognitive impairment: a follow-up study. Lancet Neurol, 5:228-34.

Heal HC, Husband HJ. 1998. Disclosing a diagnosis of dementia: Is age a factor? Aging Ment Health, 2:144-50.

Holroyd S, Turnbull Q, Wolf AM. 2002. What are patients and their families told about the diagnosis of dementia? Results of a family survey. Int $J$ Geriatr Psychiatry, 17:218-21.

Husband HJ. 2002. Diagnostic disclosure in dementia: An opportunity for intervention? Int J Geriatr Psychiatry, 15:544-7.

Hux MJ, O’Brien BJ, Iskedjian M, et al. 1998. Relation between Alzheimer's disease and costs of caring. CMAJ, 159:457-65.

Jha A, Taber N, Orrell M. 2001. To tell or not to tell - comparison of older patients' reaction to their diagnosis of dementia and depression. Int $J$ Geriatr Psychiatry, 16:879-85.

Johnson H, Bouman WP, Pinner G. 2000. On telling the truth in Alzheimer's disease: A pilot study of current practice and attitudes. Int Psychogeriatr, 12:221-9.
Jolley DJ, Benbow SM. 2000. Stigma and Alzheimer's disease: Causes, consequences and a constructive approach. Int J Clin Pract, 54:117-19.

Joosten-Wein Banningh L, Vernooij-Dassen M, Rikkert MO, et al. 2008. Mild cognitive impairment: coping with an uncertain label. Int $J$ Geriatr Psychiatry, 23:148-54.

Kahana E, Galper Y, Zilber N, et al. 2003. Epidemiology of dementia in Ashkelon: The influence of education. J Neurol, 250:424-8.

Kluger A, Ferris SH, Golomb J, et al. 1999. Neuro-psychological prediction of decline to dementia in nondemented elderly. $J$ Geriatr Psychiatry Neurol, 12:168-79.

Kral VA. 1962. Senescent forgetfulness: Benign and malignant. CMAJ, 86:257-60.

Lachman ME, Weaver S, Bandura M, et al. 1992. Improving memory and control beliefs through cognitive restructuring and self-generated strategies. J Gerontol, 47:293-9.

Leifer BP. 2003. Early diagnosis of Alzheimer's disease: Clinical and economic benefits. J Am Geriatr Soc, 51:281-8.

Levy R. 1994. Aging-associated cognitive decline. Int Psychogeriatr, 5:1-5.

Maguire CP. 2002. Telling the diagnosis of dementia: Consider each patient individually. Int Psychogeriatr, 14:123-6.

McKitrick LA, Camp CJ, Black FW. 1992. Prospective memory intervention in Alzheimer's disease. J Gerontol, 47:337-43.

Morris JC. 2006. Mild cognitive impairment is early-stage Alzheimer disease. Arch Neurol, 63:15-16.

Palmer K, Winblad B. 2007. Mild cognitive impairment: continuing controversies. Nat Clin Pract Neurol, 3:62-3.

Petersen RC. 1995. Normal aging, mild cognitive impairment, and early Alzheimer's disease. Neurology, 1:326-44.

Petersen RC. 2004. Mild cognitive impairment as a diagnostic entity. J Intern Med, 256:183-94.

Petersen RC. 2007. Mild cognitive impairment: current research and clinical implications. Semin Neurol, 27:22-31.

Petersen RC, Doody R, Kurz A, et al. 2001. Current concepts in mild cognitive impairment. Arch Neurol, 58:1985-92.

Petersen RC, Morris JC. 2003. Clinical features. In: Petersen RC, (ed) Mild cognitive impairment: Aging to Alzheimer's disease. New York: Oxford Press, pp. 15-39.

Petersen RC, Smith GE, Waring SC, et al. 1999. Mild cognitive impairment: Clinical characterization and outcomes. Arch Neurol, 56:303-8.

Pinner G, Bouman WP. 2002. To tell or not to tell: On disclosing the diagnosis of dementia. Int Psychogeriatr, 14:127-37.

Portet F, Ousset PJ, Visser PJ, et al.; the MCI Working Group of the European Consortium on Alzheimer's Disease (EADC) 2006. Mild cognitive impairment (MCI) in medical practice: a critical review of the concept and new diagnostic procedure. Report of the MCI Working Group of the European Consortium on Alzheimer's Disease. JNNP, 77:714-18.

Post SG. 2000. The Moral Challenge of Alzheimer's Disease. London: Johns Hopkins University Press.

Post SG, Whitehouse PJ. 1995. Fairhill Guidelines on ethics of the care of people with Alzheimer's disease: A clinical summary. J Am Geriatr Soc, 43:1423-9.

Price JL, Morris JC. 1999. Tangles and plaques in nondemented aging and 'preclinical' Alzheimer's disease. Ann Neurol, 45:358-68.

Pucci E, Belardinelli N, Borsetti G, et al. 2003. Relatives' attitudes towards informing patients about the diagnosis of Alzheimer's disease. $J$ Med Ethics, 29:51-4.

Ritchie K, Artero S. 2001. Classification criteria for mild cognitive impairment: A population-based validation study. Neurology, 56:37-42.

Rosenberg PB, Johnston D, Lyketsos CG. 2006. A clinical approach to mild cognitive impairment. Am J Psychiatry, 11:1884-90.

Sandman CA. 1993. Memory rehabilitation in Alzheimer's disease: Preliminary findings. Clin Gerontol, 13:19-33.

Scogin F, Bienias JL. 1988. A three-year follow-up of older adult participants in a memory-skills training program. Psychol Aging, 3:334-7.

Seidler A, Bernhardt T, Nienhaus A, et al. 2003. Association between the psychosocial network and dementia - a case-control study. J Psychiatr Res, 37:89-98. 
Talassi E, Guerreschi M, Feriani M, et al. 2007. Effectiveness of a cognitive rehabilitation program in mild dementia (MD) and mild cognitive impairment (MCI): a case control study. Arch Gerontol Geriatr, S1:391-9.

Taylor DH, Sloan FA. 2000. How much do persons with Alzheimer's disease cost Medicare? J Am Geriatr Soc, 48:639-46.

Treves T, Chapman J, Bornstein NM, et al. 1996. ApoE E4 in age-related memory complaints and Alzheimer's disease. Eur J Neurol, 3:515-8.

Van Uffelen JG, Him A Paw MJM, Hopman-Rock M, et al. 2007. The effect of walking and vitamin B supplementation on quality of life in community-dwelling adults with mild cognitive impairment: a randomized, controlled trial. Qual Life Res, 16:1137-46.

Verghese J, Lipton RB, Katz MJ, et al. 2003. Leisure activities and the risk of dementia in the elderly. N Engl J Med, 348:2508-16.

Vernooij-Dassen MJ, Van Hout HP, Hund KL, et al. 2003. Information for dementia patients and their caregivers: What information does a memory clinic pass on, and to whom? Aging Ment Health, 7:34-8.

Wadley VG, Haley WE. 2002. Diagnostic attribution versus labeling: Impact of Alzheimer's disease and major depression diagnoses on emotions, beliefs, and helping intentions of family members. J Gerontol, 56B:244-52.

Werner P. 2000. Assessing the effectiveness of a memory club for elderly persons suffering from mild cognitive deterioration. Clin Gerontol, 22:3-14.

Werner P. 2001. A review of instruments for assessing cognitive functioning in the elderly population. Gerontology, 43-9.
Werner P. 2003. A support group for elderly persons in early stages of dementia: An evaluative research. Final report presented to the Israeli Alzheimer's Association, Israel.

Werner P. 2004. Perceptions about memory problems and help seeking in elderly persons: A qualitative analysis. Clin Gerontol, 27:19-30.

Werner P. 2005. Lay persons' beliefs about mental health: where is age and where is Alzheimer's disease? Int Psychogeriatr, 17:371-82.

Werner P, Davidson M. 2004. Emotional reactions to individuals suffering from Alzheimer's disease: Examining their patterns and correlates. Int J Geriatr Psychiatry, 19:391-7.

Whitehouse PJ. 2007. Mild cognitive impairment: a confused concept? Nat Clin Pract Neurol, 3:62-3.

Whitehouse PJ, Juengst ET. 2005. Antiaging medicine and mild cognitive impairment: Practice and policy issues for geriatrics. J Am Geriatr Soc, 53:1417-22.

Wimo A, Winblad B. 2003. Pharmacoeconomics of mild cognitive impairment. Acta Neurol Scand, 179:94-9.

Winblad B, Palmer K, Kivipelto M, et al. 2004. Mild cognitive impairment - beyond controversies, towards a consensus: report of the International Working Group on Mild Cognitive Impairment. J Intern Med, 256:240-6.

Yale R. 1995. Developing Support Groups for Individuals with Early-Stage Alzheimer's Disease. Baltimore, MD: Health Professions Press.

Yesavage JA, O'Hara R, Kraemer H, et al. 2002. Modeling the prevalence and incidence of Alzheimer's disease and mild cognitive impairment. J Psychiatr Res, 36:281-6. 